\title{
20. Kulturgeschichte der Neuzeit und des Altertums:
}

\author{
Egon Friedell
}

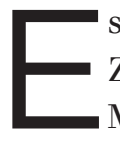

s war der Wiener Egon Friedell, seines Zeichens Kabarettist und Schauspieler bei Max Reinhardt, der es unternahm, im Ton eines geistvollen Charmeurs eine «ulturgeschichte der Neuzeit> zu erzählen.»² In drei starken Bänden, die zwischen 1927 und $193^{1}$ bei

C.H.Beck erschienen, wurde die «Krisis der europäischen Seele» vom Spätmittelalter bis zum Ersten Weltkrieg dargestellt. Der erste Band behandelte Renaissance und Reformation, der zweite Barock und Rokoko, Aufklärung und Französische Revolution, der dritte Romantik und Liberalismus, Imperialismus und Impressionismus.

\section{Der geistreiche Dilettant}

Egon Friedell, 1878 in Wien als Egon Friedmann geboren, schuf kein historisches opus magnum, sondern präsentierte ein grandioses Feuilleton, das nicht aus den Quellen, sondern aus der gelehrten Literatur schöpfte. Geistreiche Essays charakterisieren Personen und Epochen. Langeweile kommt auf keiner Seite auf, davor bewahrt den Leser der allgegenwärtige Witz des Verfassers. Friedell schätzte die anekdotische Verdichtung und die paradoxe Zuspitzung. Das Genie ist «das Geschöpf seiner Zeit», aber «die Zeit ist ganz und gar die Schöpfung des großen Mannes». ${ }^{3}$ Das Treibende in der Weltentwicklung, so fasste er Hegels dialektische Methode zusammen, ist der Widerspruch. ${ }^{4}$

Akademisches Spezialistentum verachtete der «berufene Dilettant» und Wiener Causeur, der von gebildeten Bürgern und nicht von verbeamteten Universitätsprofessoren gefeiert werden wollte. Doch was war ihm «Kulturgeschichte»? Friedell lehnte die «stumpfe und taube Registrierung von Schlachten und diplomatischen Lügen» ab und verfocht «eine Art geistiger Kostümgeschichte», «eine Geschichte der Ideen», «die die innere Seele der einzelnen Zeitalter waren, sie trugen und bewegten und ihnen den äuße- 
ren Körper bauten, der dann in Kunst und Religion, in Wissenschaft und Philosophie, in Wirtschaft und Recht und im ganzen Mienenspiel des täglichen Lebens in die Erscheinung trat». ${ }^{5}$ Das bereits 1919 formulierte Programm realisierte Friedell konsequent in seiner «Kulturgeschichte der Neuzeit», die Kulturgeschichtsschreibung zur Historiographie kat' exochen machte.

In idealistischer Tradition rückte Friedell das Individuum in das Zentrum der Darstellung, das den historischen Prozess vorantreibt, dem aber der göttliche Weltplan verborgen bleibt. Ranke irrte darin, als er glaubte, die historische Wahrheit erkennen zu können. Und mit ihm irrte die ganze Historikerzunft, die Theodor Lessing nicht gelesen oder, noch schlimmer, nicht verstanden hatte. Geschichte war logificatio post festum: «Jedes Zeitalter hat ein bestimmtes nur ihm eigentümliches Bild von allen Vergangenheiten, die seinem Bewusstsein zugänglich sind.» ${ }^{6}$ Geschichte war folglich die «fortschreitende Uminterpretierung der Vergangenheit», und ebendiese Erkenntnis begründete die Überlegenheit des Dichters über den zünftigen Gelehrten, wie Friedell in Übereinstimmung mit Spengler glaubte. ${ }^{7}$ Die «faktensammelnde Quellenforschung» lief ins Leere, so lautete die antihistoristische Botschaft der «Kulturgeschichte», denn es gab keine unstrittigen Tatsachen, sondern die historischen Fakten wurden «erst durch die Idee geschaffen, durch eine intuitive, schöpferische, poetische Erkenntnis «erschaut». Nicht die analytische Rekonstruktion von Kausalzusammenhängen wurde gefordert, sondern «durch anschauliche Konstruktion» sollten Analogiebeziehungen aufgezeigt werden. ${ }^{8}$ Friedell unterwarf seine Geschichtsschreibung nicht den Regeln der Rationalität, sondern dem Diktat der Ästhetik und immunisierte sie eben dadurch gegen die Kritik der Wissenschaft. Also bemühte er astrologische Spekulationen, um den drohenden Sieg des Bolschewismus vorherzusagen. ${ }^{9}$

Das Werk ist ein typisches Produkt der «Theaterhaftigkeit» der Weimarer Kultur, ${ }^{10}$ das durch die grandiose Collagetechnik des Autors besticht, der andere plagiierte und sich selbst zitierte. ${ }^{11}$ Später bezeichnete sich der Verfasser in schöner Selbsterkenntnis als «legitimen Plagiator» und «vorsichtigen Abschreiber».12 Der Einfluss von Nietzsche und Spengler ist offenkundig. Was Sokrates für die griechische Aufklärung, Shakespeare für die englische Renaissance, Voltaire für die französische und Lessing für die deutsche Aufklärung war, das war Nietzsche für die Gegenwart. ${ }^{13}$ Nietzsche sei allerdings an seiner Philosophie zugrunde gegangen. «In seinen letzten Schriften verwirrte sich dieser edle und kräftige Geist. Er wurde, so kann man wenigstens allenthalben vernehmen, von Größenwahn erfasst. 
Er hielt sich nämlich für Friedrich Nietzsche.» ${ }^{14}$ Auf Spenglers «Untergang des Abendlandes» bezog sich Friedell expressis verbis in der Einleitung: «Selbstverständlich hat auch Spengler nicht bloß aus dem Zeitbewusstsein geschöpft, sondern sich auch seine Vorgänger [...] zunutze gemacht. Dasselbe Recht nimmt auch die nachfolgende Darstellung für sich in Anspruch, nur dass sie in der beneidenswerten Lage war, auch schon Spengler mit abschreiben zu können.» ${ }^{15}$ Friedells Fortsetzung implizierte indes die Überwindung des Spengler'schen Kulturpessimismus. Jedes Zeitalter war zwar nicht mehr unmittelbar zu Gott, verkörperte aber einen «bestimmten Naturgedanken» und eine «eigentümliche Weltansicht». Den «Sinn des Universums» und

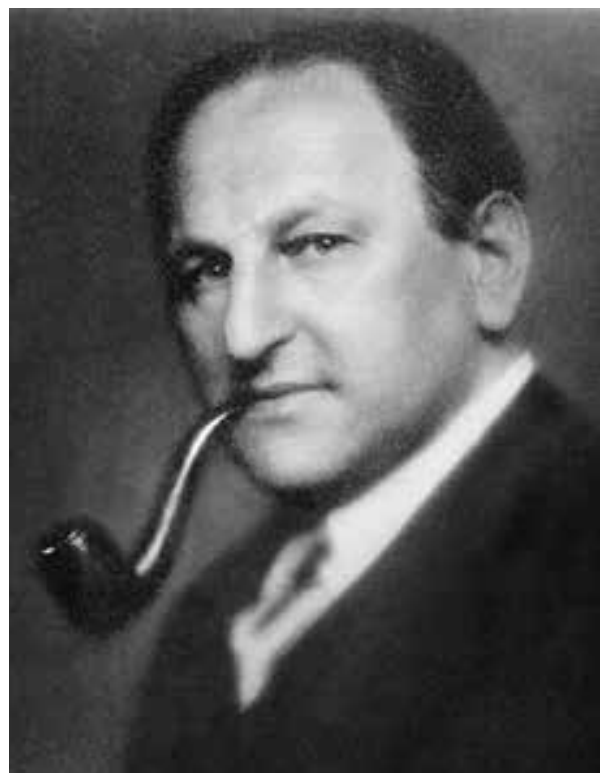

Egon Friedell «das immanente Göttliche» konnte Spengler, der «Atheist, Agnostiker, verkappte Materialist» nicht erblicken. ${ }^{16}$ «Das Abendland wird untergehen, aber nur, soweit es von Spengler ist», ${ }^{17}$ wird Friedell keine zehn Jahre später bemerken: Spengler war gestern, Friedell ist heute.

Doch auch Friedell, der konvertierte Jude, war elitär und antidemokratisch, aristokratisch und antisemitisch. An die Schauspielerin Lina Loos schrieb er, seine «Kulturgeschichte der Neuzeit» sei ein «betont anti‘modernes〉, antikapitalistisches, antitechnisches, antirationalistisches, kurz antiangelsächsisches Werk». ${ }^{18}$ Er verehrte «zwei Säulenheilige des neueren Antisemitismus», Paul de Lagarde und Houston Stewart Chamberlain, den einen als einen «der wenigen Christen, die im neunzehnten Jahrhundert gelebt haben», den anderen als «gedankenreichen Kulturphilosophen». ${ }^{19}$ Da verschlug es auch nichts, dass er Homosexuelle nicht mochte: «Jene ganze fixe Idee des Klassizismus» wird von ihm auf «die sexuelle Perversion eines deutschen Provinzantiquars» zurückgeführt, hinter dem sich niemand anderes als Johann Joachim Winckelmann verbarg. ${ }^{20}$ 
Das Buch hatte in der Tat das Zeug zum Bestseller. Aber sein Potential wurde zunächst verkannt. Dass Egon Friedell es bei C.H.Beck verlegte, war nur dem Desinteresse anderer Verlage zu verdanken. Friedell wollte 1925 seine «Kulturgeschichte der Neuzeit» bei dem renommierten Berliner Ullstein Verlag veröffentlichen. Der erste Band der «Kulturgeschichte» war bereits gedruckt, als Ullstein kalte Füße bekam. Man zweifelte offenbar in letzter Minute daran, ob Friedell, der «Kabarettist», überhaupt in der Lage sei, ein seriöses kulturhistorisches Werk zu verfassen. ${ }^{21}$ Friedell warb für sich und sein Projekt, wollte aber seine «Kulturgeschichte» entgegen den Wünschen des Verlags nicht illustrieren. Ullstein wiederum insistierte, dass Friedell alle geplanten Bände vorlegen müsse, bevor man den ersten binde und ausliefere. Es dauerte nicht lange, da ließ Friedell seinen sarkastischen Kommentaren die Drohung folgen, einen Prozess anzustrengen. Doch den Mediengiganten Ullstein konnte er nicht einschüchtern. Nachdem ein Jahr verstrichen war, ohne dass die Ausgabe des ersten Bandes nähergerückt wäre, half der Journalist Paul Wiegler, Leiter der Romanabteilung im Ullstein Verlag und bestens im Buchhandel vernetzt, «seinem> Autor und machte sich, da er nach wie vor an das Werk glaubte, auf die Suche nach einem neuen Verleger. Erst sagten, um Friedell zu zitieren, eine ganze Reihe von «Judenverlagen» ab, und dann konnte ein christlicher Verlag «aus finanztechnischen Gründen» das Projekt nicht unmittelbar realisieren. Schließlich bot Wiegler das Buch C.H.Beck an, und Heinrich Beck soll nach einem kurzen Blick in das Manuskript begeistert gewesen sein. Er zerstreute die Bedenken gegen ein Werk von «überspitzter Subjektivität und heiterer Leichtigkeit», das «Weder zur Schulwissenschaft, der der Verlag sonst zu dienen bestrebt war», passte, «noch zu dem schweren Ernst der Spengler'schen Geschichtsphilosophie». ${ }^{22}$ Offenbar hatte auch Friedell selbst im Verlag vorgesprochen, wo sich Walther Eggert-Windegg nachdrücklich für die «Kulturgeschichte» aussprach und auch August Albers das Werk «mit großer Wärme» empfahl. ${ }^{23}$

Im Februar 1927 wurde der Vertrag unterzeichnet. Als Honorar waren zwölfeinhalb Prozent vom Ladenpreis der broschierten Exemplare vorgesehen. Beim ersten Erscheinen eines Bandes wurde jeweils das Honorar für 2000 Exemplare voraushonoriert. Zum Ausgleich der verlorenen Auslagen, die C.H.Beck durch die besonderen Umstände der Übernahme der Buchblöcke des ersten Bandes von Ullstein entstanden waren, stellte 
Friedell einen Betrag von 1000 Mark zur Verfügung, indem er von dem fälligen Honorar bei Ausgabe des ersten und zweiten Bandes jeweils auf 500 Mark verzichtete. ${ }^{24}$ Im Mai desselben Jahres erschien der erste Band der «Kulturgeschichte der Neuzeit» im Verlag C.H.Beck. Der Band war, wie es Friedell wünschte, unbebildert. Wie schon zuvor bei Oswald Spengler und Albert Schweitzer war es der Kairos, dem der Verlag es zu verdanken hatte, dass jetzt die dritte große kulturgeschichtliche Darstellung der Weimarer Republik bei C.H.Beck veröffentlicht wurde. Systematisch warb man für Friedells Buch. Schon Mitte Juli 1927 teilte August Albers dem ihm gut bekannten Rezensenten Otto Heuschele mit, er freue sich, dass dieser eine Besprechung des Friedell-Buches verfassen werde: «Seit Spenglers Werk sind von keinem mehr so viele Rezensionsexemplare verlangt worden wie von diesem. Ich habe schon ein ganz dickes Kuvert voll von solchen Anforderungen, denen wir aber nicht mehr entsprechen können.»25

Im Herbst 1928 wurde der zweite Band veröffentlicht, der die Zeit vom Dreißigjährigen bis zum Siebenjährigen Krieg umfasste. Friedell hatte zügig das Manuskript fertiggestellt; jetzt plagte ihn die Sorge, er könne sich schlecht verkaufen, da der Absatz stockte. Heinrich Beck beruhigte ihn: «Das erscheint mir ganz natürlich und wiederholt sich bei allen solchen mehrbändigen Werken.» Die meisten Interessenten würden nicht gleich alle Bände auf einmal kaufen, sondern zunächst einmal nur den ersten Band. «Freilich begnügt sich mancher Leser dann damit, den ersten Band gelesen zu haben. Ein zweiter Band wird deshalb immer etwas in der Auflagenziffer hinter dem ersten zurückstehen. Dies war auch bei dem Spengler'schen Werk der Fall.» ${ }^{26}$ Beck verglich Friedells Werk inzwischen mit dem Erfolgsbuch «Der Untergang des Abendlandes» des Vorzeigeintellektuellen der Konservativen Revolution. Zumindest der Verleger zweifelte nicht mehr an dem Erfolg von Friedells «Kulturgeschichte». Er beruhigte den Autor zunächst mit dem Hinweis auf den Weihnachtsverkauf, der den Absatz ankurbeln werde, und verwies auf das Interesse angelsächsischer Verlage, die sich um das Übersetzungsrecht des Werkes bemühten. Nochmals verglich er Friedells und Spenglers Werk: «Was eine französische Übersetzung betrifft, so neige ich da zu großer Zurückhaltung. Wir konnten uns bis jetzt auch noch nicht zu einer französischen Übersetzung des ‘Untergangs des Abendlandes〉 entschließen, trotz vieler Angebote. Die Bücher sind in Frankreich so teuer und die Honorare, die prozentual zum Buchpreis vergütet werden, so niedrig, dass weder für den Autor noch für den Verlag etwas herausspringt. Dagegen macht sich naturgemäß die Konkurrenz einer billigen französischen Ausgabe im 
Ausland sehr fühlbar und verringert dadurch den Absatz des deutschen Werkes beträchtlich. Ein angemessenes Honorar von einem französischen Verleger zu erzielen, ist so gut wie ausgeschlossen.» ${ }^{27}$ Tatsächlich erschienen Übersetzungen auch später nur in nordeuropäischen und angelsächsischen Ländern. Eine geplante italienische Ausgabe ließ sich ebenfalls nicht realisieren.

Abschließend erinnerte der Verleger an den noch ausstehenden dritten Band der «Kulturgeschichte» und gab seiner Hoffnung Ausdruck, dass ein mehrmonatiges Engagement des Autors und Schauspielers Friedell auf der Bühne in Berlin seiner Arbeit an dem Buch keinen Abbruch tun werde. Seine Befürchtungen waren unbegründet. Der dritte Band erschien 1931. Schon in den ersten Jahren wurden 2700 Exemplare verkauft, insgesamt 81 ooo Einzelbände. ${ }^{28} 1930$ erschien die erste amerikanische Ausgabe. Das Feuilleton feierte das Buch und seinen Verfasser. ${ }^{29}$

Heinrich Beck zögerte nicht, die Zusammenarbeit mit dem erfolgreichen Autor, mit dem er inzwischen auch privat verkehrte, fortzusetzen. Diskutiert wurden seit Ende $193^{2}$ eine «Kulturgeschichte des Altertums» und ein «Konversationslexikon». ${ }^{30}$ Als Heinrich Beck erfuhr, dass das Wörterbuch mit kulturgeschichtlichen Lemmata einen Umfang von 1500 Seiten annehmen würde, sah er seine ursprünglichen Vorbehalte bestätigt und riet im März 1933 dringend, die «Kulturgeschichte des Altertums» zu schreiben. Doch der überbordende Umfang war nur ein Grund für Heinrich Becks Entscheidung. Die ironischen Einträge des Lexikons waren nach der «Machtergreifung» der Nationalsozialisten politisch viel zu brisant: «Es ist nunmehr tatsächlich zweifelhaft geworden, ob man als Verleger nicht einem Verdikt anheimfällt, wenn man ein weltanschauliches Lexikon herausbringt, dessen Sätze sich mit der Weltanschauung des Nationalsozialismus nicht in allen Punkten decken», schrieb Heinrich Beck am 27. März 1933. ${ }^{31}$ Zwei Tage später kam es zum Abschluss des Vertrags über die «Kulturgeschichte des Altertums». Das Werk sollte aus zwei Bänden bestehen. Wiederum wurde ein Honorar von zwölfeinhalb Prozent «Vom gehefteten Ladenpreis» festgesetzt. Friedell erhielt allerdings eine monatliche Vorauszahlung von 6 oo Mark. Als Honorarvorausvergütung wurden maximal 14,400 Mark pro Band vereinbart. ${ }^{32}$ Von dem «Konversationslexikon» war künftig keine Rede mehr. 
Doch in Deutschland war das «Reich des Antichrist» angebrochen, in dem, wie Friedell bitter kommentierte, «jede Regung von Noblesse, Frömmigkeit, Bildung, Vernunft von einer Rotte verkommener Hausknechte auf die gehässigste und ordinärste Weise verfolgt» wurde. ${ }^{33} \mathrm{Er}$ zog sich mit seiner Haushälterin Herma Kotab in sein Haus bei Kufstein zurück, wo er an der «Kulturgeschichte des Altertums» zu arbeiten begann. Heinrich Beck besuchte ihn, auch wenn die Zusammenkünfte beschwerlich waren, da Hitler die Grenze zu Österreich hatte sperren lassen. Man traf sich klandestin im Garten eines Zollaufsehers an der bayerisch-österreichischen Grenze und in einem Wirtshaus neben dem Grenzposten. Doch auch der freie Devisenverkehr zwischen Österreich und dem nationalsozialistischen Deutschland wurde unterbunden. 1934 konnten die aus der «Kulturgeschichte der Neuzeit» erwirtschafteten Honorare nicht mehr ausbezahlt, sondern Friedell nur noch gutgeschrieben werden. Das Guthaben sollte spätestens dann zur Auszahlung gelangen, wenn «die Honorare aus dem Verkauf der Kulturgeschichte des Altertums die auf dieses Werk geleisteten Vorauszahlungen überschreiten». Heinrich Beck war noch guten $\mathrm{Mu}-$ tes, dass Verlag und Autor mit dem Werk Gewinn machen würden. ${ }^{34}$ Bei der Abwicklung der Geschäfte und der Kontrolle der Zahlungen stand Friedell mit Erwin Goldarbeiter ein Vertrauter Max Reinhardts zur Seite. ${ }^{55}$

Ende 1934, erreichte das erste Kapitel der «Kulturgeschichte des Altertums» über «Das Geheimnis Ägyptens» den Verleger. Heinrich Beck war höchst angetan. «Die Kulturgeschichte Ägyptens» habe er mit größtem Genuss gelesen, ließ er den Autor am 16. Januar 1935 wissen. Friedell habe mit diesem Kapitel eine ganz neue Form der Darstellung gefunden. Diese übertreffe die «Kulturgeschichte der Neuzeit» an «Gemeinverständlichkeit und Volkstümlichkeit des Erzählertons, ohne deshalb auf feine Lichter und Pikanterien der Gedankenführung irgendwie zu verzichten». Friedell solle bitte diesen Stil durch das ganze Buch durchhalten, so dass die Darstellung des Altertums «nicht schulhaft belehrend, sondern wirklich volkstümlich unterhaltend» sei. Er glaube, dass Frauen geradezu begeistert von diesem Werk sein müssten. Vom Standpunkt des Verlegers sei diese Darstellung nachgerade als ideal zu bezeichnen. ${ }^{36}$

Heinrich Beck mahnte zum zügigen Schreiben. Friedell dürfe sich nicht den Alttestamentler Albrecht Alt zum Vorbild nehmen, dessen Band über Syrien, Palästina und Arabien, der als Teil der «Kulturgeschichte des Alten Orients» im Rahmen des «Handbuchs der Altertumswissenschaft» erschei- 
nen solle, sich aber immer noch im Druck befinde. Alt sei aufgrund der politischen Unruhen der letzten Jahre «nicht mehr zur inneren Sammlung und wissenschaftlichen Arbeit» gekommen.

Der Brief enthielt zudem persönliche Mitteilungen, die eindrücklich zeigen, wie vertraut der Umgang zwischen Verleger und Autor war. Die «Neujahrswünsche mit Bildnis» hätten ihn sehr gefreut, bekundete Heinrich Beck. «Verzeihen Sie mir, dass ich sie erst heute erwidere. Ich wollte Ihnen auch irgendetwas Bildliches bieten. Da aber eine Fotografie von mir nach keiner Richtung hin besondere Bedeutung hätte und auch keine existiert, die ich verbreitet sehen möchte, so schicke ich Ihnen ein Bild meines Sohnes bei seinen ersten Versuchen, sich im Sinn seiner Vorfahren als Verleger zu betätigen. Da ich Ihnen schon solche Intimitäten meines Familienlebens preisgebe, so darf ich erwähnen, dass am Heiligen Abend meine Familie durch ein drittes Kind, ein Töchterchen, vermehrt wurde.» ${ }^{37}$

Doch rasch wich die Zuversicht, dass das Buch bei C.H.Beck erscheinen würde. «Die unerbittlichen Maßnahmen der Reichsschrifttumskammer gegen das «nicht-arische> Schrifttum» ließen den Verleger, wie es in der Verlagsgeschichte von 1963 rückblickend hieß, daran zweifeln, ob er selbst «mit einem politisch so neutralen Gegenstand» wie einer «Kulturgeschichte des Altertums» noch «durchkomme». ${ }^{38}$ Im Laufe des Jahres 1935 fasste Heinrich Beck den Entschluss, von dem Projekt zurückzutreten. Es war abzusehen, dass «jüdisches» Schrifttum indiziert werden würde. In Bayern hatte die «Politische Polizei» ein «Verzeichnis der polizeilich beschlagnahmten und eingezogenen sowie der für Leihbüchereien verbotenen Denkschriften» herausgegeben. Auf Reichsebene wurde am 25. April 1935 die «Anordnung über schädliches und unerwünschtes Schrifttum» erlassen, die fünf Tage später im «Börsenblatt für den Deutschen Buchhandel» zu lesen war. Die Reichsschrifttumskammer baute in Zusammenarbeit mit dem Reichsministerium für Volksaufklärung und Propaganda systematisch die Instrumente zur Buchzensur aus. Ende 1935 wurde die erste Liste «des schädlichen und unerwünschten Schrifttums» vorgelegt. ${ }^{39}$ Nachdem Heinrich Beck mit Spengler bereits einen umstrittenen Autor im Programm hatte, wollte er offenbar nicht auch noch mit einem jüdischen Schriftsteller die Parteistellen provozieren. Zudem war offenkundig, dass mit indizierten Titeln kein Geld mehr zu verdienen war. Die Wege von C.H.Beck und Egon Friedell trennten sich. Aber die Loyalität zu seinem Autor veranlasste Heinrich Beck, Friedell eine Perspektive zu eröffnen, die es ihm gestattete, seine Bücher weiterhin zu veröffentlichen. Also handelte Heinrich Beck im Dezember 1935 mit dem Phaidon Verlag in Wien einen Vertrag 
aus, der die Vereinbarung enthielt, dass die Rechte an der «Kulturgeschichte des Altertums» an den Autor zurückfielen. Damit nicht genug. Heinrich Beck hatte erkannt, dass auch die «Kulturgeschichte der Neuzeit» früher oder später der Zensur anheimfiele. Deshalb räumte er Friedell das Recht ein, eine Lizenz für eine Volksausgabe zu vergeben. Vertraglich verzichtete die C.H. Beck'sche Verlagsbuchhandlung auf einen weiteren Neudruck der «Kulturgeschichte der Neuzeit», «sofern die Volksausgabe zu gegebener Zeit, spätestens im Laufe des Jahres 1939 tatsächlich erscheint. Die Rechte der C.H. Beck'schen Verlagsbuchhandlung, auch die Genehmigung von Übersetzungslizenzen usw. bleiben unberührt. Ist die Volksausgabe der «Kulturgeschichte der Neuzeit〉 vergriffen und erscheint binnen zwei Jahren keine Neuauflage, so fallen die Verlagsrechte an der «ulturgeschichte der Neuzeit im ganzen Umfang wieder an die C.H. Beck'sche Verlagsbuchhandlung zurück.» ${ }^{40}$ Schon am 3. Dezember 1935 hatte man Erwin Goldarbeiter gebeten, darauf hinzuwirken, dass Egon Friedell seine Bücher künftig nicht mehr über C.H.Beck, sondern über Phaidon bestellen sollte, da der Wiener Verlag «die wesentlich vorteilhaftere Beschaffungsmöglichkeit» böte. ${ }^{41}$

Am 8. Mai 1936 starb Oswald Spengler. Egon Friedell ließ, von der Nachricht ergriffen, Heinrich Beck einige Zeilen zukommen. Der Verleger antwortete am 28. Mai 1936: «Mein großer Autor Spengler hat keine sehr freundliche Presse gehabt, als er so überraschend die Augen schloss. Deshalb ist es mir wirklich ein gewisser Trost, wenn mir von privater Seite mitgeteilt wird, dass man sein Scheiden als einen Verlust empfindet.» Beck wollte dann wissen, ob die Wiener Presse irgendwelche ausführlicheren Nekrologe gebracht habe. In den skandinavischen Ländern sei Spengler sehr achtungsvoll behandelt worden. In der Schweiz hingegen sei man wegen seiner antidemokratischen Haltung auf ihn «böse». «Offenbar sind es in der ganzen Welt Durchschnitts-Journalisten gewesen, die man zu diesem Zweck herangezogen hat.» Dann beklagte er den Tod seiner beiden verdienten Mitarbeiter, Walther Eggert-Windegg und August Albers. Albers, der «Propagandaleiter», sei «auf recht tragische Weise aus dem Leben geschieden». Er fügte hinzu, für das «interne Gesicht» des Verlags bedeute dieses Frühjahr «einen recht entscheidenden Einschnitt, der nach außen hin durch den Tod Spenglers symbolisiert wird». Auch der Umstand, dass Friedells «Kulturgeschichte des Altertums» nun in einem anderen Verlag erscheine, werde in diese Richtung wirken, er habe das Gefühl, «irgendwie von vorne anfangen zu müssen». ${ }^{42} \mathrm{Um}$ sein Unternehmen im neuen Deutschland positionieren zu können, musste er sich mit den Machthabern 
arrangieren. Die Absage an das Verlagsprojekt einer «Kulturgeschichte des Altertums», das der jüdische Autor Egon Friedell übernommen hatte, gehörte zu dieser Neuorientierung. Heinrich Beck litt unter dieser Entwicklung und klagte über seine angeschlagene Gesundheit.

\section{Verfolgt und entrechtet}

Mit dem Inhaber des Phaidon Verlags, Béla Horovitz, hatte man darüber hinaus vereinbart, die «Kulturgeschichte des Altertums» zunächst in Österreich zu verlegen. Heinrich Beck war optimistisch, dass der Phaidon Verlag die geeigneten Wege finden werde, Friedells neues Buch auch in Deutschland abzusetzen. Er gehe davon aus, schrieb er an Friedell, dass sich die Presse wahrscheinlich jetzt «beträchtlichste Zurückhaltung» auferlegen werde, das Buch aber dank dem Glanz seiner Darstellung «rasch von Mund zu Mund empfohlen» werde. Die Widmung an den norwegischen Literaturnobelpreisträger und Hitlersympathisanten Knut Hamsun erachtete er als einen ausgezeichneten Gedanken. ${ }^{43}$ Tatsächlich erschien der erste Teil der «Kulturgeschichte des Altertums», der den Untertitel «Leben und Legende der vorchristlichen Seele» trug und Ägypten sowie den Alten Orient behandelte, im November 1936 nicht in Österreich, sondern im Zürcher Helikon-Verlag, der zur Phaidon-Gruppe gehörte. Der Schweizer Verlag unterhielt Vertretungen in Berlin und Leipzig. Das «Börsenblatt für den Deutschen Buchhandel» weigerte sich, das Buch anzukündigen. De facto war es damit in Deutschland verboten. ${ }^{44}$ Inzwischen war Friedells Name aus «Kürschners Deutschem Literaturkalender» getilgt worden. Und Heinrich Beck selbst schrieb keinen Brief mehr an seinen Autor.

Friedell sagte sich in seiner «Kulturgeschichte des Altertums» von der Religion seiner Väter los, pries den christlichen «Ketzer» Marcion, der das Alte Testament nicht als christliche Offenbarungsschrift anerkannt hatte, und sah in einem von allen jüdischen Elementen gereinigten Christentum die «einzige Religion, die diesen Namen verdient». ${ }^{45}$ Wie er sich selbst wahrnahm und wie er von anderen wahrgenommen werden wollte, kleidete er in ein Zitat, das er Houston Stewart Chamberlains antimodernem und antisemitischem Bestseller «Die Grundlagen des neunzehnten Jahrhunderts» entnommen hatte: «Andrerseits ist es sinnlos, einen Israeliten echtester Abstammung, dem es gelungen ist, die Fesseln Esras und Nehemias abzuwerfen, in dessen Kopf das Gesetz Mose und in dessen Herzen die Verachtung andrer keine Stätte mehr findet, einen Juden zu nennen.» ${ }^{46}$ 
Doch in Deutschland wollte man die Schriften des «Juden» Egon Friedell nicht mehr lesen. Am 8. Februar 1937 holte die Reichsschrifttumsstelle beim Reichsministerium für Volksaufklärung und Propaganda bei der Reichsschrifttumskammer in Berlin Informationen über den Autor des ersten Teiles der «Kulturgeschichte des Altertums» ein. Die Reichsschrifttumsstelle bat zu prüfen, «inwieweit der Vertrieb dieses Buches in Deutschland erwünscht sein kann». Zugleich wies man darauf hin, dass der Verfasser «vor der Machtübernahme abwechselnd in Österreich und Deutschland anzutreffen» gewesen sei, «seit der Machtergreifung aber seinen Wohnsitz ausschließlich in Österreich» habe. Keine seiner früheren Schriften sei laut der ersten Liste des schädlichen und unerwünschten Schrifttums von 1935 und den bisher veröffentlichten Nachträgen verboten, und seine dreibändige «Kulturgeschichte», die zwischen 1928 und 1931 erschienen war, werde nach wie vor durch den deutschen Buchhandel vertrieben. ${ }^{47}$

In der Reichsschrifttumskammer kam es daraufhin zu hektischer Betriebsamkeit. Egon Friedell war, da er in Österreich wohnte, bisher nicht erfasst worden. Am 19. April 1937 verlangte man von der C.H.Beck'schen Verlagsbuchhandlung je ein Exemplar der im Verlag erschienenen Bücher Friedells und bat mitzuteilen, was über die Person des Autors bekannt sei. Am 26. April 1937 antwortete die Berliner Abteilung des Verlags dem Präsidenten der Reichsschrifttumskammer: «Der Verfasser des in den Jahren 1927 bis $193^{1}$ bei uns erschienenen Werkes Kulturgeschichte der Neuzeit), drei Bände, von dem wir wunschgemäß gleichzeitig ein Exemplar übersenden, ist von Beruf Schauspieler und Schriftsteller. Er ist Österreicher und lebt in Wien. Abgesehen von dem bei uns erschienenen Werk hat er vor allem Bühnendichtungen und Übersetzungen veröffentlicht, die Sie in Kürschners Literaturlexikon aufgeführt finden. Im Januar dieses Jahres ist im Helikon-Verlag in Zürich der erste Band einer «ulturgeschichte des Altertums> erschienen. Die Familienverhältnisse von Herrn Friedell sind uns im Einzelnen nicht näher bekannt. Soweit wir unterrichtet sind, ist er von einer elterlichen Seite nicht Arier. Heil Hitler!» ${ }^{48}$

Am 7. Mai bestätigte die Kammer, dass die Vermutung, der Verfasser sei «nicht Arier», zutreffe: Es handle sich «um den Juden Egon Friedländer [sic]». Im Verwaltungsgang der nationalsozialistischen Bürokratie war aus Egon Friedells «bürgerlichem Namen» Friedmann «Friedländer» geworden. Dem Verlag wurde mitgeteilt, dass die Kammer annehme, C.H.Beck werde unter diesen Umständen von einer Neuauflage des Werkes absehen; zugleich bat sie um Mitteilung, wie viele rohe, broschierte und gebundene Exemplare der vorliegenden Auflage noch vorrätig seien und 
wie man sich den Vertrieb der Restbestände vorstelle. Am zo. Juni antwortete wiederum die Berliner Abteilung, dass man nicht die Absicht habe, eine neue Auflage des Buches zu veranstalten, da die Vorräte noch etwa 2000 Exemplare pro Band betrügen. «In der Propaganda haben wir uns seit Jahren außerordentlich zurückgehalten. Unsere Absicht war, uns auf die Ausführung einlaufender Bestellungen zu beschränken. Wir haben auch schon erwogen, die vorhandenen Bestände einer Wiener Auslieferungsstelle zu übergeben.» Die Kammer legte am 16. Juli 1937 dem Verlag nahe, die Angelegenheit sei am besten dadurch aus der Welt zu schaffen, dass «die Bestände von Friedells Kulturgeschichte der Neuzeit einer Wiener Auslieferungsstelle übergeben würden». ${ }^{49}$

Am 25. Oktober informierte C.H.Beck die Reichsschrifttumskammer, «dass die Gesamtauslieferung» der «Kulturgeschichte der Neuzeit» «etwa ab Mitte November des Jahres» von einer Wiener Firma übernommen werde. Doch schon am 16. November musste man zurückrudern: Die Übertragung der Auslieferung an das österreichische Unternehmen war gescheitert. «Die Ausführung des Planes würde den Absatz des Werkes in Deutschland und in der Schweiz so gut wie völlig unterbinden und uns einer sehr empfindlichen wirtschaftlichen Schädigung aussetzen, die wir zu vermeiden trachten müssen.» Der Verlag beantragte, die Auslieferung des Werkes im Inland in der bisherigen Weise weiterhin zuzulassen. Selbstverständlich werde man sich - «wie schon in den letzten Jahren» «jeder Propaganda für das Werk in Deutschland enthalten und die Bestände nur stillschweigend ausverkaufen, ohne das Werk neu aufzulegen. Wir werden daneben bemüht bleiben, eine Lösung zu finden, die, ohne uns allzu großen wirtschaftlichen Schädigungen auszusetzen, die Abwicklung der Angelegenheit beschleunigt.» Den Verkauf der Vertragsrechte ins Ausland und die Rückgabe dieser Rechte an den Autor wollte man, so argumentierte der Verlag gegenüber der Reichsschrifttumskammer, aus zwei weiteren Gründen zunächst vermeiden: Sobald C.H.Beck völlig auf die Verlagsrechte verzichte, werde das Werk ohne Weiteres in einem österreichischen oder schweizerischen Verlag neu entstehen und sehr viel nachhaltiger verbreitet werden, und die ausländischen Übersetzungsrechte des Werkes, die dann auch an den Autor zurückfallen würden, brächten noch ganz annehmbare Honorare und damit für Deutschland Devisenbeträge, die man «retten» könne. In etwa zwei Jahren würden diese Eingänge im Wesentlichen wohl erschöpft sein..$^{50}$ C.H.Beck versuchte, durch wirtschaftliche Argumente die Politik der Kammer zu unterlaufen.

Die Reichsschrifttumskammer leitete am 23. November 1937 dieses 
Schreiben abschriftlich an das Reichsministerium für Volksaufklärung und Propaganda weiter, das schon mehrfach Stellungnahmen in dieser Sache angemahnt hatte. Die vom Verlag angeführten Gründe wurden von der Kammer nicht anerkannt, «da es ja gerade der Sinn der geplanten Maßnahme war, dass der Absatz des Werkes in Deutschland möglichst unterbunden wurde». Unter keinen Umständen könne es befürwortet werden, dass eine von einem «Juden» verfasste «Kulturgeschichte» heute noch in Deutschland vertrieben werde. ${ }^{51}$ Das Reichsministerium für Volksaufklärung und Propaganda bestätigte am 27. Januar 1938 dem Geschäftsführer der Reichsschriftumskammer, Wilhelm Ihde, man sehe «keinerlei Notwendigkeit für die weitere Verbreitung der Kulturgeschichte der Neuzeit von Egon Friedländer [sic], genannt Friedell». Das Buch werde in die «Liste des schädlichen und unerwünschten Schrifttums» aufgenommen. Dem Verlag wurde von der Kammer am 31. Januar 1938 mitgeteilt, dass die weitere Verbreitung des Werkes nach \$ 1 der Anordnung über schädliches und unerwünschtes Schrifttum vom 25. April 1935 untersagt sei. Ein Durchschlag der Entscheidung der Kammer - «in Übereinstimmung mit dem Herrn Reichsminister für Volksaufklärung und Propaganda» - ging an die Geheime Staatspolizei.

C.H.Beck versuchte nun $\mathrm{zu}$ retten, was noch $\mathrm{zu}$ retten war. Am 8. Februar 1938 bestätigte der Verlag den Erhalt des Schreibens vom 31. Januar. Man werde selbstverständlich die darin auferlegten Bedingungen erfüllen und jede weitere Verbreitung des Werkes in Deutschland einstellen. Man bitte jedoch, «die Weggabe des Werkes ins Ausland» zu genehmigen. Die Eingabe des Verlags leitete die Reichsschrifttumskammer am 14. Februar 1938 befürwortend an das Reichsministerium für Volksaufklärung und Propaganda weiter und verwies auf die überzeugenden wirtschaftlichen Gründe. Dann spitzten sich die Dinge dramatisch zu. Am 21. Februar 1938 erschien ein Beamter der Gestapo in den Münchner Verlagsräumen, um die Bestände des Werkes zu beschlagnahmen. Die Bücher sollten der Makulierung zugeführt werden, «und zwar zu einem Bruchteil des sonst für Makulierung üblichen Gegenwertes». Per Eilboten bat C.H.Beck die Reichsschrifttumskammer, dass die Gestapo über die Bestände nicht eher verfügen solle, als über den Antrag vom 8. Februar entschieden sei. Man wolle die Vorräte geschlossen ins Ausland verkaufen, wie dies die Kammer bereits 1937 im Prinzip genehmigt habe. Nochmals wurden die Gründe genannt, die für eine solche Entscheidung sprächen: Eine Neuauflage des Werkes im Ausland würde verzögert werden, und man könne mit dem Eingang von Devisen rechnen. Stimme die Kammer dem Vorschlag nicht 
zu, würden diese Maßnahmen «eine besondere Härte» für den Verlag bedeuten, «da das Honorar für die noch übrigen Vorräte schon vor vielen Jahren an den Autor ausbezahlt wurde. Wir bitten Sie, bei Ihrer Entscheidung zu beachten, dass es sich einerseits um einen ausländischen Verfasser, andererseits nicht um eine deutsche, sondern um eine allgemeine Kulturgeschichte handelt.» ${ }^{52}$

Zwei Tage nachdem die Gestapo in den Verlagsräumen die «Kulturgeschichte der Neuzeit» beschlagnahmen wollte, ging Friedell der letzte Brief aus München zu. Dem «sehr verehrten Herrn Doktor» wurde mitgeteilt, dass der kürzlich erteilte Auftrag, ein Exemplar der «Kulturgeschichte der Neuzeit» an einen Oberstudienrat zu versenden, nicht ausgeführt werden konnte, weil die Auslieferung des Werkes von der Reichsschrifttumskammer untersagt worden sei. «Wir wollten Sie, ehe eine endgültige Entscheidung gefallen war, nicht in Unruhe stürzen. Nun ist uns mitgeteilt worden, dass das Verbot der Auslieferung Ihres Werkes als endgültig zu betrachten ist.» Ein Vertrag auf Verwertung der gedruckten und gebundenen Vorräte im Ausland sei zurzeit noch nicht zustande gekommen. «Sobald wir Näheres wissen, werden wir Ihnen darüber gesonderte Mitteilung machen.» Die schlechte Nachricht überbrachte «mit besten Empfehlungen» nicht der Verleger, sondern sein Mitarbeiter Georg Sund;53 der promovierte Germanist, der bereits 1924, in den Verlag eingetreten und zwölf Jahre lang die Herstellungsabteilung des Verlags geleitet hatte, war seit Albers' und Eggert-Windeggs Tod im Jahre 1936 für das literarische und geisteswissenschaftliche Programm verantwortlich. ${ }^{54}$

Der Verlag erreichte durch die Intervention in der Tat, dass die Vernichtung der Bücher verhindert werden konnte. Selbst das Propagandaministerium verschloss sich den Argumenten nicht und erklärte am 7. März 1938 sein Einverständnis für den Verkauf ins Ausland. Dann machte die Zeitgeschichte die Verlagsstrategie zunichte. Am 12. März 1938 überschritten deutsche Truppen die Grenze nach Österreich. Am Tag darauf verkündete Adolf Hitler den «Anschluss» seiner Heimat an das Großdeutsche Reich. Am 16. März, gegen zehn Uhr abends, sprang Egon Friedell aus dem Fenster seiner im dritten Stock gelegenen Wiener Wohnung in den Tod. Zwei junge SA-Männer sollen an der Wohnungstür nach dem «Jud Friedell» gefragt haben. ${ }^{55}$ Am 11. April 1938 meldete die Gestapo der Reichsschrifttumskammer: «Unter Hinweis auf den nunmehr erfolgten Anschluss Österreichs an das Reich wurde der Inhaber der C.H. Beck'schen Verlagsbuchhandlung Dr. Beck befragt, ob er eine weitere Möglichkeit der Veräußerung der Rechtsbestände ins Ausland ins Auge gefasst habe. Er hat 
erklärt, dass für ihn nunmehr kein Interesse an einem Verkauf bestände. Ich bitte um Zustimmung, dass die beschlagnahmten Bücherbestände endgültig vernichtet werden.» Ende April des Jahres wurde die Makulierung von 2000 Exemplaren der «Kulturgeschichte der Neuzeit» in die Wege geleitet. ${ }^{56}$ Damit verschwand Egon Friedells Erfolgsbuch aus dem Verlagsprogramm.

\section{Der Streit um das Erbe}

In der Verlagsgeschichte von 1963 bemerkte Heinrich Beck, dass es nach dem Krieg gelungen sei, «die Verlagsrechte an diesen Büchern zurückzuerwerben und vor allem die Kulturgeschichte der Neuzeit» nochmals zu einem glanzvollen Erfolg zu führen». ${ }^{57}$ Damit umschrieb er elegant einen schweren Konflikt um die Rechte an den Werken Egon Friedells, der nach dem Ende des Zweiten Weltkriegs zwischen C.H.Beck und dem Phaidon Verlag ausgebrochen war. Nach dem Anschluss Österreichs hatte Phaidon seinen Sitz nach Großbritannien verlegt. 1947 erschien in diesem Verlag die zweite Auflage der «Kulturgeschichte Ägyptens und des Alten Orients», und 1949 sollte die «Kulturgeschichte Griechenlands» folgen, deren beiden ersten Kapitel bereits 1940 in Oslo in einer norwegischen Übersetzung veröffentlicht worden waren.

Am 19. Februar 1948 eröffnete Béla Horovitz, der Inhaber der Phaidon Press in London, die Korrespondenz mit der Mitteilung, man sei sehr überrascht, im «Börsenblatt für den Deutschen Buchhandel» zu sehen, dass der Münchner Verlag die «Kulturgeschichte der Neuzeit» wieder angekündigt habe. Darin sehe man einen Eingriff in die eigenen Rechte, denn Egon Friedell selbst habe die Rechte an seinem Werk dem Phaidon Verlag übertragen. Heinrich Beck war über den Brief so erschüttert, dass er ihn in seinem Tagebuch erwähnte. ${ }^{58}$ Doch er beharrte auf seinem Recht an dem Autor, dessen Werk er im Spruchkammerverfahren als «essentiell demokratisch» gepriesen und das ihm als Beweis gedient hatte, um seine Distanz zu den Nationalsozialisten herauszustellen: Er habe zugunsten seines «jüdischen Autors Egon Friedell noch nach der Machtergreifung Hitlers und in voller Erkenntnis der drohenden Gefahren einen Vertrag unterzeichnet», durch den er sich verpflichtet habe, Friedell «vier Jahre lang eine Rente von monatlich 6 oo Mark zu zahlen; durch diese Zahlungen sollte ihm ermöglicht werden, ein neues Werk zu schreiben».59 Dass er Ende 1935 von dem Vertrag zurückgetreten war, erwähnte er nicht. 
Am 2o. September 194,8 legte Horovitz detailliert seine Auffassung der Rechtslage dar: Der Phaidon Verlag verfüge über die Rechte sowohl an der «Kulturgeschichte der Neuzeit» als auch an den beiden Teilen der «Kulturgeschichte des Altertums», der «Kulturgeschichte Ägyptens» und der «Kulturgeschichte Griechenlands». Horovitz bot lediglich Vertriebsrechte an allen Titeln in Deutschland an. Der Gewinn sollte geteilt werden und der Vertrag zunächst für fünf Jahre gelten. Die Verhandlungen um das «schwere Sorgenkind» belasteten Heinrich Beck. ${ }^{60}$ Am 13. November 1948 ließ er Horovitz wissen, dass die Verträge über die «Kulturgeschichte der Neuzeit» zwischen dem Phaidon Verlag und den Erben Egon Friedells befristet gewesen seien. Überdies habe Phaidon sie nicht erfüllt, denn es sei eine «Volksausgabe» vereinbart worden, die Phaidon aber nicht innerhalb der Vertragsfrist herausgebracht habe. In München schlug man deshalb einen gemeinsamen Vertrieb aller Werke vor: C.H.Beck übernehme Deutschland und Österreich, Phaidon fielen alle anderen Länder zu. Die Verlagsrechte an der «Kulturgeschichte der Neuzeit» sollten bei Beck, die an den beiden anderen Bänden bei Phaidon verbleiben. ${ }^{61}$

Am 14. Dezember 1948 wies Horovitz den Vorwurf der Nichterfüllung des Vertrages über die «Kulturgeschichte der Neuzeit» scharf zurück. Auf die Behauptung, der Vertrag habe eine befristete Vergabe vorgesehen, ging man in London nicht weiter ein. Man bestand vielmehr darauf, dass C.H.Beck die Rechte nicht mehr habe, sondern allenfalls zurückerhalten könne. Ein Konsens war zunächst nicht zu erzielen. Da Heinrich Beck den Anspruch des Phaidon Verlags nicht anerkannte, dass die deutschsprachigen Verlagsrechte ausschließlich dem Londoner Verlag, der auch eine Niederlassung in Zürich hatte, zustünden, versuchte er Ende 1949 den Konflikt durch den Vorschlag zu lösen, eine Feststellung über die Rechtslage solle ganz unterlassen werden. Phaidon bewegte sich jedoch nicht. Strittig blieb die Frage, ob der Londoner Verlag aus dem 1935 abgeschlossenen Vertrag seine Rechte an der «Kulturgeschichte der Neuzeit» ableiten könne, obwohl die einbändige «Volksausgabe» angesichts der Wirren der Zeitläufte erst 1947 veröffentlicht worden war. Die Angelegenheit wurde noch dadurch verkompliziert, dass Friedell offenbar später einen weiteren Vertrag geschlossen hatte, der es Phaidon gestattete, das Erscheinen der Volksausgabe zu verschieben. Offenbar hatte der Autor in diesem Punkte Zugeständnisse gemacht, die im Widerspruch zu seinen Verpflichtungen gegenüber Beck standen. Im Fortgang der Verhandlungen drohte Horovitz, man würde Regressansprüche gegen die Erben stellen, würde man in einem allfälligen Rechtsstreit unterliegen. Angesichts dieser Aussichten 
gelang es Heinrich Beck, Friedells Erbin Herma Kotab und Walther Schneider, ihren Vertrauten, auf seine Seite zu ziehen, obschon sie zuvor Béla Horovitz unterstützt hatten. ${ }^{62}$ Nun vertraute Herma Kotab in dem Honorarstreit auf Heinrich Becks Rat. ${ }^{63}$

Am 21. März 1950 kam es zu einem Treffen zwischen Heinrich Beck, Béla Horovitz und Herma Kotab in Zürich. Man begann den Verhandlungsmarathon mit einem gemeinsamen Abendessen. Mehrtägige Gespräche folgten, in deren Verlauf es gelang, Horovitz von einer Klage abzubringen und einen Kompromiss zu schließen. ${ }^{64}$ Da aber erst im Juli die Devisen genehmigt wurden, die es C.H.Beck erlaubten, 84,0o Exemplare von Friedells «Kulturgeschichte Griechenlands» für 26520 Schweizer Franken von Phaidon zu kaufen, ${ }^{65}$ konnten die Verträge über die «Kulturgeschichte des Altertums» und die «Kulturgeschichte der Neuzeit» erst im August paraphiert werden. Die Vergleiche, die geschlossen wurden, trugen dem komplexen Gegenstand Rechnung und sicherten in den nächsten Jahrzehnten die Verlagsrechte und die Gewinnbeteiligung des Phaidon Verlags an den Werken. Man erkannte an, dass beide Verlage aufgrund älterer Verträge Rechte an dem Werk «Kulturgeschichte der Neuzeit» hatten, und einigte sich, dass das Verlagsrecht an der deutschsprachigen Ausgabe ihnen gemeinsam $z u$ stand. Die Verlagsrechte an der «Kulturgeschichte der Neuzeit» sollten jedoch allein durch C.H.Beck, die an der «Kulturgeschichte des Altertums» gemeinsam durch beide Verlage ausgeübt werden. C.H.Beck beteiligte Phaidon mit siebeneinhalb Prozent vom Ladenpreis der gehefteten und viereinhalb Prozent vom Ladenpreis der gebundenen Exemplare der «Kulturgeschichte der Neuzeit». Zudem kam man darin überein, eine Volksausgabe des Werkes zu veranstalten. Weitere Auflagen der «Kulturgeschichte Ägyptens und des Alten Orients» und der «Kulturgeschichte Griechenlands» wurden in München hergestellt und vertrieben. An dem Reingewinn beteiligte C.H.Beck den Phaidon Verlag mit fünfzig Prozent. Später änderte man diese Bestimmung: Phaidon erhielt eine Tantieme von fünf Prozent des Ladenpreises der gehefteten Exemplare; ab der zweiten Auflage waren es sechs Prozent. ${ }^{66}$

Die nachfolgenden Verhandlungen bezogen sich auf die Versuche, Friedells Werke einerseits bei Buchgemeinschaften und andererseits als preisgünstige Taschenbuchausgaben herauszubringen. Die Demokratisierung des Buchmarkts in der jungen Bundesrepublik erfasste auch Egon Friedells «Kulturgeschichten» aus den zwanziger und dreißiger Jahren. Phaidon drängte Heinrich Beck immer wieder, einen Buchclub für das Projekt zu interessieren, um entsprechende Honorareinnahmen zu erzielen, und 
setzte ihn auch dadurch unter Druck, dass man die Möglichkeit eigener Aktivitäten in Deutschland andeutete. ${ }^{67}$ Doch den literarischen Massenmarkt wollte C.H.Beck selbst bestellen. 196o brachte man mit der Zustimmung des Phaidon Verlags anstelle der dreibändigen «Kulturgeschichte der Neuzeit» eine einbändige ungekürzte Sonderausgabe heraus, die rasch hohe Verkaufszahlen erzielte. 1960 erschien das 33 . bis 53 . Tausend, 1961 das 54 . bis 79 . Tausend, 1984 das 136 . bis 146 . Tausend. ${ }^{68}$ Die «Kulturgeschichte der Neuzeit» erschien zuletzt 2012 in neuer Ausstattung in «Becks Historischer Bibliothek». Die Gesamtauflage beträgt heute mehr als 200 ooo Exemplare.

Doch Heinrich Beck wollte mit Friedells Büchern auch an dem dynamisch expandierenden Markt für Taschenbücher partizipieren. C.H.Beck gehörte zu den elf Gesellschaftern des neu gegründeten Deutschen Taschenbuch Verlags (dtv), der am zo. November 1960 ins Handelsregister eingetragen wurde und ab September 1961 mit monatlichen Taschenbuchauslieferungen an die Öffentlichkeit trat. Allerdings fehlte den Verträgen, die bisher mit dem Phaidon Verlag und der Erbin Friedells abgeschlossen worden waren, eine Regelung, auf welcher Grundlage die Einnahmen für Taschenbuchlizenzen unter den drei Beteiligten zu verteilen wären. Heinrich Beck ergriff hier die Initiative und schlug einen Festbetrag für Phaidon im unteren Pfennigbereich vor. In diesem Segment musste mit abgesenkten Gewinnspannen operiert werden. Am 16. Mai hakte Phaidon nach und wollte die Drittelung der Lizenzgebühren durchsetzen. Heinrich Beck insistierte auf einem festen Niedrighonorar. Denn nur «mit der Autorenseite müsste unser Verlag wohl fifty-fifty abrechnen, wie es dem ursprünglichen Verlagsvertrag entspricht». Beck begründete seine Ablehnung einer Drittelung unter anderem damit, dass der Münchner Verlag seit Jahrzehnten «die ganze Last der Propaganda und des Vertriebes getragen» habe. Phaidon stimmte schließlich zu ${ }^{69}$ Friedell gehörte mit zu den ersten Autoren, die Heinrich Beck für eine Veröffentlichung im Deutschen Taschenbuch Verlag als geeignet erachtete. 1961 wurde bei dtv der Abschnitt «Aufklärung und Revolution» aus der «Kulturgeschichte der Neuzeit» zum Ladenpreis von DM 3,6o veröffentlicht: C.H.Beck erhielt pro verkauftem Exemplar neunzehn Pfennige, von denen fünfzig Prozent an die Erben gingen. Aus dem eigenen Honorar überwies Beck dem Phaidon Verlag vier Pfennige. ${ }^{70}$ Egon Friedell schrieb, mit anderen Worten, auch Taschenbuchgeschichte. 